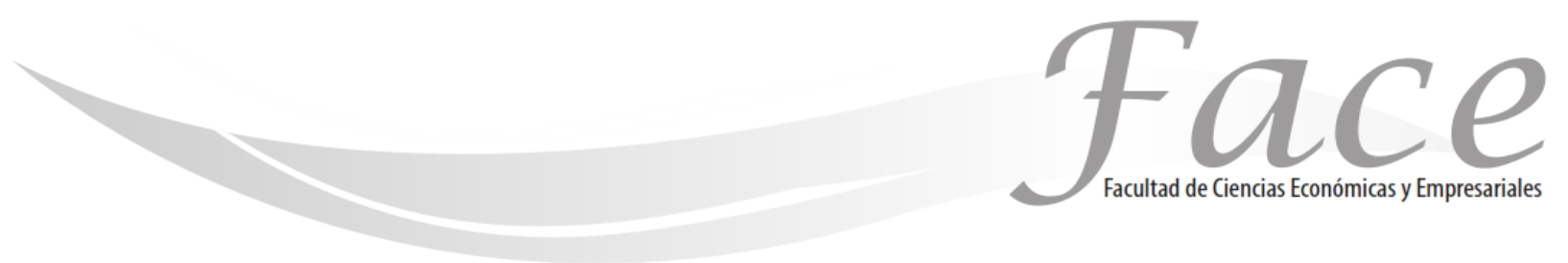

ISSN Impreso: 1794-9920

ISSN Electrónico: 2500-9338

Volumen 17-N²

Año 2017

Págs. 55 - 67

\title{
OUTPLACEMENT COMO HERRAMIENTA ESTRATÉGICA PARA REDUCIR MOMENTOS DE INCERTIDUMBRE EN ORGANIZACIONES: UNA PERSPECTIVA REFLEXIVA Y ATEMPORAL
}

\author{
Dr. Denny J. Pereira B. * \\ Enlace ORCID: http://orcid.org/ 0000-0002-1725-0888 \\ Dr. Doile E. Rios P. ** \\ Enlace ORCID: http://orcid.org/0000-0002-1974-6924
}

Fecha de Recepción: febrero 12017

Fecha de Aprobación: mayo 32017

\section{Resumen:}

La presente investigación tuvo como principal propósito Comprender el outplacement como herramienta estratégica para la reducción de momentos de incertidumbre en organizaciones. La misma se sustentó teóricamente en autores como Licht, A. (2003), Muñiz (2008), Carmona (2009), León (2006) entre otros. Epistemológicamente, la investigación tiene un abordaje cualitativo, con un método de investigación de tradición cualitativa Fenomenológica. Los tripulantes clave fueron seleccionados de diferentes organizaciones con diversas filosofías y atención, teniendo como única similitud su razón social inicial, donde la constitución de estas fue bajo una economía privada y hoy en día son empresas adscritas al gobierno nacional. En cuanto a la técnica de recolección se empleó la observación participante y la entrevista en profundidad, constituida por una guía semiestructurada y como instrumento se recurrió a la grabadora portátil y los registros descriptivos. Las técnicas de análisis se desarrollaron a través de la categorización, estructuración, triangulación y teorización, en base al método de microanálisis propio de la teoría fundamentada propuesto por Strauss y Corbín (2002) para el tratamiento de la data. Entre los resultados destacados se pudo constatar la necesidad de construir una teoría sustantiva que explique el outplacement como herramienta estratégica para la reducción de momentos de incertidumbre en organizaciones. Se concluyó que en las instituciones existen ficciones de técnicas o programas para un proceso adecuado de transición y/o cambio de filosofía organizacional. Se recomienda el outplacement como herramienta estratégica en las organizaciones, en las que la productividad no es el único fin, sino que es relevante los procesos de manera de obtener en los empleados desvinculados esa aceptación, encontrando esta situación como una oportunidad de cambio; que no necesariamente tiene que ser limitante, por el contrario puede ser vista como una opción de mejoramiento a nivel personal, laboral y profesional.

Palabras Claves: Outplacement, Desvinculación Asistida, Cambio Organizacional, Filosofía Organizacional, Incertidumbre. 
* Licenciado en Educación y Licenciado en Filosofía, con títulos de Maestría en Ciencias de la Educación mención Gerencia Educativa, Doctorado en Ciencia de la Educación y Certificación Postdoctoral en Gerencia de la Educación Superior. doilerios@gmail.com

** Venezolano, Nacido en Maracaibo, Estado Zulia Licenciado en contaduría Pública egresado de La Universidad del Zulia (LUZ), Magister en Gerencia Empresarial / Especialización en Docencia para Educación Universitaria / Doctor en Ciencias Gerenciales egresado de la Universidad Rafael Belloso Chacín (URBE), Investigador activo de la Universidad Rafael Belloso Chacín dennypereira@gmail.com

\title{
OUTPLACEMENT AS A TOOL TO REDUCE STRATEGIC UNCERTAINTY IN ORGANIZATIONS MOMENTS A THOUGHTFUL AND TIMELESS PERSPECTIVE
}

\begin{abstract}
The present research was to understand the main purpose outplacement as a strategic tool to reduce times of uncertainty in organizations. It was based theoretically on authors such as Licht, A. (2003), Muñiz (2008), Carmona (2009), Leon (2006) among others. Epistemologically, research has a qualitative approach with a qualitative research method Fenomenológica tradition. The key crew members were selected from different organizations with different philosophies and attention, with the only similarity initial company name, where the constitution of these was under a private economy and today are companies affiliated to the national government. As for the art collection participant observation and in-depth interview, consisting of a semi-structured guide and as an instrument to portable recorder and descriptive records was used was used. Analysis techniques were developed through categorization, structuring, triangulation and theorizing, based on the method itself microanalysis grounded theory proposed by Strauss and Corbin (2002) for the treatment of data. Among the outstanding results it was found the need to build a substantive theory to explain the outplacement as a strategic tool to reduce times of uncertainty in organizations. It was concluded that there are fictions of techniques or programs to a proper process of transition and / or change in organizational philosophy in institutions. outplacement It is recommended as a strategic tool in organizations where productivity is not the only purpose, but is relevant processes way to get in unlinked employees that acceptance, finding this as an opportunity for change; that does not necessarily have to be limited, on the other hand can be seen as an option for improving professional staff level, employment and.
\end{abstract}

Keywords: Outplacement, Untying Assisted, Organizational Change, Organizational Philosophy, Uncertainty. 


\title{
EXPLORAMENTO COMO HERRAMIENTA ESTRATÉGICA PARA REDUCIR MOMENTOS DE INCERTIDUMBRE EN ORGANIZACIONES: UNA PERSPECTIVA REFLEXIVA Y ATEMPORAL
}

\author{
Resumo
}

\begin{abstract}
Esta pesquisa foi outplacement entender o propósito primário como uma ferramenta estratégica para reduzir tempos de incerteza nas organizações. A mesma teoria foi baseada em autores como Licht, A. (2003), Muñiz (2008), Carmona (2009), Leon (2006), entre outros. Epistemologicamente, a pesquisa tem uma abordagem qualitativa com um método de pesquisa qualitativa fenomenológica tradição. Os membros principais da tripulação foram selecionados de diferentes organizações com diferentes filosofias e atenção, com o nome da empresa inicial única semelhança, onde a constituição destes estava sob uma economia privada e hoje são empresas afiliadas ao governo nacional. Quanto à observação participante técnica de coleta e entrevista em profundidade, constituído por um instrumento semi-estruturado como o gravador portátil e registros descritivos ele foi usado foi usado. técnicas de análise foram desenvolvidas através de categorização, estruturação e triangulação teorização, com base no método de microanálise própria teoria fundamentada proposta por Strauss e Corbin (2002) para o tratamento dos dados. Entre os excelentes resultados verificou-se a necessidade de construir uma teoria substantiva para explicar o outplacement como uma ferramenta estratégica para reduzir tempos de incerteza nas organizações. Concluiu-se que há ficções de técnicas ou programas para um processo adequado de transição e / ou mudança de filosofia organizacional em instituições. outplacement é recomendado como uma ferramenta estratégica nas organizações onde a produtividade não é o único efeito, é processos relevantes para que os funcionários se desligado em que a aceitação, encontrando isso como uma oportunidade para a mudança; que não tem necessariamente de ser uma limitação, por outro lado pode ser visto como uma opção para melhorar 0 nível profissional, de emprego e..
\end{abstract}

Palavras-chave: Outplacement, Desvinculação de energia, mudança organizacional, filosofia organizacional, incerteza. 


\section{INTRODUCCIÓN:}

Las organizaciones se encuentran conformadas por grupos de humanos complejos que actúan dentro de un contexto de tiempo-espacio de manera concreta y deliberadamente constituido para la realización de fines y necesidades específicas. Ello significa satisfacer necesidades sociales, relacionadas con un comportamiento moralmente responsable y a una contribución en los asuntos que los individuos por sí solos no pueden abordar.

Pero la actuación de las empresas no solo se manifiesta en la actividad comercial 0 en la prestación de un bien 0 servicio. Los efectos de sus acciones son juzgados en diversos planos, como por ejemplo los culturales, sociales, políticos, entre otros. También es el caso de la discontinuidad de la relación laboral dispuesta por la empresa, que es el objeto de interés en este estudio.

Si bien es cierto, la pérdida del empleo produce en forma inmediata un problema económico, también provoca dificultades familiares, hostilidad en los que lo rodean, pérdida de prestigio y de estima en sí mismo además de un entorno lleno de incertidumbre. Por todo esto, una herramienta como el Outplacement es tan importante como la política de selección de personal. Ambos son momentos extremos en la vida laboral de un trabajador. La euforia, al ser elegido entre los postulantes, genera un sentimiento de "el mejor". La desvalorización, al ser elegido para abandonar la empresa, produce un sentimiento de ser "el peor".

La realidad en el ámbito laboral no refleja estos sentimientos extremos, por ello deberá ser responsabilidad de la empresa el tratamiento de la desvinculación laboral que pueda minimizar los momentos de incertidumbre generados dentro del seno organizacional. Las políticas de personal que piensan fundamentalmente en el apoyo a aquel colaborador que necesita una ayuda especial por la situación difícil que tiene que afrontar debido a los efectos de una decisión no tomada por él mismo, son simultáneamente un mensaje que la organización le da a todos aquellos que continúan brindando sus servicios a la misma.

Una manera de contemplar la situación al talento humano que la organización decide desvincular es mediante la implementación de la herramienta de outplacement, que ofrecen al personal afectados estrategias para enfrentar de mejor manera la situación desvinculativa, sea en relación de dependencia 0 no, de acuerdo con las aptitudes personales e intereses particulares sobre el porvenir colectivo.

Dicha herramienta, que desarrollaré en el estudio en profundidad, tiende a suavizar los efectos de la dolorosa situación que deben enfrentar las personas desvinculadas. Es por ello, que en este estudio, se buscó estudiar el
Outplacement como herramienta estratégica para reducir momentos de incertidumbre en organizaciones, para lo cual, la investigación estuvo compuesta por constructos teóricos de los distintos autores que dan explicación sobre el outplacement así como lo concerniente a la incertidumbre en el ámbito gerencial, todo adaptado a esas organizaciones en proceso de cambio teniendo como punto inicial las cualidades 0 rasgos percibidos por el investigador durante la recolección de la data y sin perder de vista los hallazgos que emergen de cada una de las entrevistas realizadas en profundidad.

Seguidamente, se realizó una aproximación teórica sustantiva y finalmente se generó el mecanismo del outplacement como una teoría emergente, donde el investigador no solo llega a alcanzar una mayor comprensión del fenómeno, sino que comparte esta comprensión con los demás, surgiendo así una teoría propia del estudio y dando postura sobre el asunto. Luego de ello, se presentan las consideraciones finales y referencias bibliográficas.

\section{MARCO TEÓRICO:}

\section{OUTPLACEMENT}

Las organizaciones están operando en tiempos impredecibles, de continuos cambios y demandas globales, pues cada día se genera una creciente presión en ellas para que éstas compitan de manera más eficiente. Buscando alinear la estrategia de idoneidad con los objetivos de negocio en las instituciones, puede ser usual encontrarse reestructuraciones así como recortes de personal para el cumplimiento de las nuevas metas.

Según Barragán (2000), el outplacement se basa en la recolocación de personas y es un servicio que consiste en un conjunto de medidas que una empresa, a través de un despacho especializado, ofrece a los empleados, ante la desvinculación de las organizaciones, donde se pone a disposición del empleado todas las herramientas para la búsqueda de un empleo. Para tratar con personas que se encuentran en plena reingeniería es preciso tener tacto, preparación, sensibilidad y un proceso estructurado o constituido.

La manera en la que actuará un ser en proceso de cambio, dependerá de cómo se realizó la acción, según el informante 1 "Resistencia esa fue mi actitud, resistencia y todo porque no me prepararon para ese cambio tan brusco, ninguno de dos patrones ni el anterior ni este nos prepararon". Sobre la base de las ideas expuestas por este informante, el investigador aporta que el no preparar adecuadamente al personal ante una restructuración, evidentemente no se puede esperar una aceptación plena 
Denny J. Pereira B.- Doile E. Rios P.

en los individuos, al contrario el clima laboral se tornará resistente a cambiar.

Según Licht (2003), el outplacement se está convirtiendo cada vez más en una herramienta popular, puesto que las fusiones, adquisiciones y reestructuraciones empresariales son algunas de las principales causas de una desvinculación. Pues este instrumento consolidado al llevarse apropiadamente a la práctica puede ayudar tanto a la organización en proceso de cambio como a los empleados afectados por ese proceso de restructuración laboral. Cada vez más las instituciones ha reflexionado considerablemente para suministrar servicios diversificados a las necesidades que demande el talento humano que posee, independientemente de su nivel jerárquico.

En este mismo orden de ideas, Sastre (1995) manifiesta el "outplacement es un servicio aportado por una empresa a uno 0 varios trabajadores despedidos, esta puede ser aplicada por la misma empresa o mediante consultores externos". Es entonces que también entre los beneficios ofrecidos por esta herramienta gerencial está el asesoramiento, la capacitación o formación y la detención necesarias de estrategias para lograr una transición de trayectoria o experiencia.

Atendiendo a estas consideraciones, dichas detención se realiza logrando buscar con ello una pronta reinserción laboral según sea el perfil de competencia, así como sus preferencias minimizando considerablemente los perjuicios tanto económicos como psicológicos que pudieran ser generados en toda situación de despido, cambio organizacional, jubilación, renuncia o cambio de patronos.

En líneas generales, establece el investigador que la desvinculación asistida permite que los empleados que afrontan una situación delicada y negativa como es el desempleo lo conviertan en una oportunidad. En la que se sientan tomados en cuenta, apoyados y logren desarrollar sus capacidades para desafiar su nueva situación, así poder mejorar sus condiciones emocionales. A la vez que las empresas conservan un equipo comprometido, responsable además poseedor de valores éticos ante sus compañeros.

\section{Filosofía organizacional}

En cuanto a esta sub categoría, se debe mencionar que para poder evolucionar, crecer, permanecer en el tiempo generando utilidades como organización se deben estar consciente de la dirección y objetivo que se quiere tomar, es decir, ya se debe tener marcado o definido la estrategia desde los cimientos u origen de la filosofía organizacional.

Al respecto, autores como Muñiz (2008) define la filosofía organizacional como el sistema de valores y creencias de una organización. Por lo tanto, la misma está compuesta por una serie de principios, que se basan en saber quiénes somos, y en quien creemos, es decir, ideas y valores; las cuales son preceptos; así como conocer cuáles son los compromisos y las responsabilidades.

Cuando se habla de filosofía organizacional, se hace alusión a la asistencia del desarrollo de una empresa junto con la visión que le puede dar cada empleador, esto va de la mano con la planeación ya que permite descubrir lo que se quiere y/o hacia donde se quiere llegar. Así mismo menciona que posee varios tipos de planeación como la estratégica que es utilizada a mediano o a largo plazo, la táctica o funcional que solo es atraída para situaciones específicas y finalmente la operativa que opera a corto plazo.

Por su parte, Pérez (2008) trata los valores, las convicciones filosóficas de los gerentes encargados de guiar a su empresa en un viaje exitoso; misión, el concepto general de su empresa; visión, cómo debe ser su empresa en el futuro, y estrategia, la dirección en que debe avanzar su empresa. Estos tienden a ser esencialmente elementos intuitivos, es decir basados en los sentimientos más que analíticos asentados en la información.

Al hacerse énfasis en lo expresado por el informante 2 "los cambios en una organización son buenos, porque cada cambio que tuvimos en la empresa privada siempre eran para mejor", para ello es necesario facultar al personal a la hora de hacer unos arreglos en la filosofía organizacional, siempre y cuando esté dirigido a colaborar positivamente con la gestión reestructuradora dominando las emociones generadoras del cambio.

De modo similar, el informante 4 relata que después de una intervención de terceros para cambiar la filosofía organizacional, se debe primero pretender eliminar la visiones mercantilistas dándole un toque más social 0 humanista; así mismo en la p. 3, fila 58 expresa: "han cambiado más que todo la ideología política y la incursión a lo político es lo que más que todo han cambiado".

Por su parte, Drucker (2005) plantea que la filosofía organizacional examina los principios fundamentales que subyacen en las estructuras de formación al igual que de actividades de las instituciones empresariales, donde ellas puedan dar respuesta práctica basadas en experiencias a cualquier conflicto surgido en la naturaleza y en el propósito del hecho empresarial. Entonces, el objeto de esta resulta atrayente para los gestores, especialistas y directivos organizacionales, pero también para el resto de la masa trabajadora, pues la filosofía organizacional aborda temas empresariales, comunicacionales, de ética, hasta de política entre otras. 
Atendiendo a estas consideraciones, evidentemente al querer cambiar una filosofía organizacional preestablecida se debe trabajar todo ese conjunto de valores, creencias, entendimientos y hasta ideologías políticas que el talento humano normalmente debe contar para el cumplimiento de los objetivos de cualquier institución, direccionados todos a ofrecer formas definidas de pensamiento, sentimiento y reacción que guían la toma de decisiones estratégicas, así como otras actividades de los colaboradores dentro de la organización.

\section{Aprendizaje organizacional}

El aprendizaje organizacional es el proceso donde las entidades bien sean grandes o pequeñas, tal vez públicas o privadas, convierten la información en conocimiento, lo propagan y explotan con el fin de desarrollar toda capacidad innovadora pero sobre todo competitiva. Hoy día constituye una poderosa herramienta para incrementar el capital intelectual de una empresa, aumentando así sus capacidades para la resolución de problemas cada vez más confusos con la intención de poder adaptarse al entorno.

Actualmente, existen numerosas definiciones al respecto y que conduce la temática. De allí, que Carrión (2005), conceptualiza el aprendizaje organizacional como el proceso mediante el cual se integran conocimientos, habilidades y actitudes para conseguir cambios o mejoras de conducta en los empleados. Coincidentemente, Folk, Sánchez y Vallbe, (2006), lo definen como el proceso mediante el cual una institución facilita la adquisición y creación de nuevos conocimientos a sus miembros ya los grupos para alcanzar con ello un conocimiento global; permitiendo entonces adoptar los cambios adecuados a las demandas del entorno empresarial.

En tal sentido, el investigador aporta que el aprendizaje organizacional involucra una serie de mecanismos utilizados como herramientas que permitan la transformación del conocimiento en aquellas personas 0 esos equipos perteneciente a la empresa para la obtención de un saber colectivo en función de las metas y objetivos de la institución.

En este orden de ideas, es acertado el planteamiento de Barnes, (2006), quien refiere que aprender es cambiar la conducta, el propósito es alcanzar una forma de comportamiento más efectiva o competente, no sólo es conocimiento, implica habilidades, valor y compromiso. En este sentido, Swieringa y Wierdsma (2007) precisan el Aprendizaje Organizacional como el cambio del comportamiento del personal de una organización.

Por su parte, el informante 2 al compartir que "el equipo que ejecutó el proceso de cambio no tenía ningún norte de cómo eran los cambios que tenían que ejecutar como administración pública, entonces desde ese momento todo fue ensayo y error", pues el informante considera que todos proceso debe estar diseñado por un método adecuado permita llegar al propósito focal de la organización esto compartiendo ideas o conversatorio, utilizados como encuentros formativos de equipos de trabajo, e incluso comunidades de aprendizaje.

Por consiguiente, el aprendizaje organizacional es representado por ese conocimiento basado en la experiencia consecuente de determinada labor desempeñada, causa en el cual la masa trabajadora de una institución poseen las competencias para intercambiar opiniones, valores, ideas y hasta creencias a través de una interacción constructiva de la práctica cotidiana en su entorno laboral, generando de manera colectiva el progreso institucional con la potenciación de una gestión basada en conocimiento.

En tal sentido, de acuerdo con lo expuesto por Senge (2005), como aporte valioso para este estudio, al exponer que en las organizaciones la función del gerente como líder empresarial, cobra significativamente una gran importancia en el proceso de aprendizaje organizacional, ya que se enfrenta ante un importante reto en la actualidad, como lo es conseguir que capital humano que integran la empresa aprendan, evolucionen profesionalmente para lograr que mejoren de manera continua para el avance institucional convirtiéndolo en resultados objetivos y medibles como aprendizaje permanentemente de la misma.

Según el investigador, esto se hace necesario en las instituciones seleccionadas para el estudio, es decir estas empresas que cambiaron su filosofía organizacional; que actualmente deben atender una población que demanda una serie de servicios específicos relacionados el bienestar social y político, requieren una atención apropiada durante su estadía en cada espacio. de modo que la interiorización de los procesos en forma de aprendizaje correspondiente a la esencia de la organización permitido al trabajador brindar una mejor atención a los usuarios con quien operan.

\section{Incertidumbre}

Las organizaciones están operando en tiempos impredecibles llenos de incertidumbre gracias a los continuos cambios que demanda el mercado global, pues se está ejerciendo una creciente presión en cada una de las empresas para que éstas compitan de manera más eficiente y puedan sobrevivir en el tiempo. Sobre este particular, Carmona (2009) indica que existe un ambiente de incertidumbre cuando falta el conocimiento seguro y claro respecto del desenlace o consecuencias futuras de alguna acción, situación o elemento patrimonial, lo que 
puede derivar en riesgo cuando se aprecia la perspectiva de una contingencia con posibilidad de generar pérdidas 0 la proximidad de un daño.

Seguidamente León (2006) opina que la incertidumbre se gestiona con más diálogo e interacción; para que la empresa pueda afrontar con éxito la complejidad del entorno competitivo cambiante debe aumentar su complejidad, aunque no tanto en su estructura (orden) como en su función y tratamiento de la información (comportamiento). Con estos parámetros, consideramos que pensar en un modelo de gestión estratégica implica pensar en un modelo de gestión de la incertidumbre para generar organizaciones flexibles y competentes.

Asimismo, expresa el informante 3 que incertidumbre ciertamente viene alimentada de inseguridades 0 confusiones, miedo a cambiar, pues lo desconocido siempre genera molestare emocionales donde la adaptación se hace difícil. Podría decirse entonces que se entiende por incertidumbre a un escenario en la cual no se conoce completamente la probabilidad de que acontezca un determinado evento o suceso.

Ahora bien, para el investigador es evidente entonces que la mayoría de las personas por no decir todas tienden a ver la incertidumbre como algo estrictamente negativo que muchas veces quieren evitar a toda costa. Cualquier cosa que rompa con la seguridad, que sea difícil de controlar detona la alerta en la persona, como si tuviera que enfrentarse a su peor enemigo. Todo este mecanismo de defensa es una lucha absurda e irracional, que únicamente desgasta al individuo puesto que la incertidumbre forma parte del día a día de cualquier ser humano y es por tanto ineludible o necesario, no podemos evitarla y mucho menos en organizaciones vanguardista.

Visto de este modo, las organizaciones están operando en tiempos impredecibles llenos de incertidumbre gracias a los continuos cambios que demanda el mercado global, pues se está ejerciendo una creciente presión en cada una de las empresas para que éstas compitan de manera más eficiente y puedan sobrevivir en el tiempo.

Según el planteamiento de Sull y Escolari (2005), la incertidumbre como punto de origen a la innovación; esta a su vez permite definir a los ambientes de incertidumbre, como aquellos donde las variables como regulaciones, tecnología, oferta - demanda, capacidad y acceso al capital cambian de manera veloz e inesperadamente, tal es la incertidumbre, que se potencia o magnifica con las interacciones entre los cambios.

Comparando las afirmaciones de los autores citados que señalan que las organizaciones en ambientes de incertidumbre se muestran a dos posibles contextos como lo son: las oportunidades de oro, referidas a las ocasiones donde una firma puede alterar de manera significativa el destino organizacional, su capacidad de crear define la consolidación de un valor futurista, un concepto muy ligado con la definición de destrucción creativa; las amenazas de muerte súbita, refiriéndose a los distintos choques presentado con el entorno comprometedor, basado en la supervivencia de la firma, determinado por ser intermitentes, impredecibles, retadoras de creación además de surtidora de valor.

Sobre la base de los planteamientos anteriores, el investigador asume que es esta la oportunidad de relacionar la red cognitiva de la combinación integradora de las variables universales y esenciales donde se entrelazan las organizaciones resilientes con la inestabilidad 0 turbulencia empresarial, señalando aspectos que logran ventajas competitivas al momento de liderar alianzas que generan espacios para adaptar estrategias empresariales a escalas globales, fundamentadas en la innovación.

\section{Cambio organizacional}

Son muchos los cambios que están ocurriendo en las organizaciones, de igual manera resulta difícil diferenciar aquello que los detona. Es por ello que toda institución no pueden conformarse con ser observadores y permitir que las cosas sucedan sin nada que puedan hacer, pues esto puede conducir inseguridad en cuanto al propio futuro.

Ahora bien, existen cambios que pueden ocurrir inesperadamente, provocando una vertiginosa inestabilidad, al respecto relata el informante 1 "primero que nada me sentí inseguro" también admitió que "hay empresas que cambian de dueño y ellos precisamente hacen todo un trabajo de adaptación de los trabajadores, precisamente para que ese cambio no sea así (...), todo fue abrupto y casi sin aviso".

Visto de este modo, el investigador aporta que lo más significativo es que si no se prepara gerencialmente al personal, muchas veces la alternativa es saber lidiar con lo ocurrido intentando sacar el mejor provecho posible a la situación, puesto que cuando la empresa pasa por un proceso de cambio, es difícil para el personal tener control de sus carreras emocionales.

Según Chiavenato (2007), "el proceso de cambio organizacional comienza cuando surgen fuerzas que crean la necesidades de establecer transformaciones en una 0 varias secciones de la organización" para tal efecto el informante 3, p. 7, fila: 246 "los cambios en una organización son buenos, porque cada cambio que tuvimos 
en la empresa privada siempre eran para mejor y se notaban se reflejaban a medida que pasaba el tiempo".

Para Fernández (2004), concibe el proceso psicológico por el que las personas deben pasar para encontrarse en concordancia con la nueva situación, es lo que se define como transición. El cambio no sucede sin este proceso donde todos los actores involucrados no ven claramente el norte de la situación, comenzado a emerger las incertidumbres y los perjuicios personales que esta nueva situación les podría traer aparejado a cada uno tales como: pérdida de poder, de status, duplicidad o sobrecargas de tareas, posibles nuevos jefes, así mismo, emergen autos cuestionamientos acerca de su capacidad, e interrogante de la más numerosas, en muchos casos justificada índole.

Arremeter a un proceso de gerencia del cambio, tal como lo expresa Denison (2006), no es una acción tan fácil como pudiera pensarse debido a una serie de elementos que lo conforma; involucrando los compendios que la integra, además de que para ello se debe estar totalmente seguro de que la organización pueda absorber adecuadamente los cambios, muy particularmente, que su talento humano puedan comprender su importancia comprometiéndolos de hecho en su desempeño laboral, teniendo en cuenta que el mismo es un proceso permanente que hay que tratarlo como tal y no como algo temporal.

Ahora bien, también es importante resaltar, gracias a las exhortaciones insistentes del autor citado que ante cualquier proceso de cambio organizacional es necesario manejar muy integradamente tanto los aspectos técnicos como los humanos, pues, sin capacidad para tratar los aspectos de gestión humana, el proceso de aceptación del cambio donde por ende la adopción de los aspectos técnicos propiamente del proceso cambio o el propósito principal del cambio organizacional, en función, resultan mucho más arduos, dificultosos y hasta pueden tener un grado de probabilidad hacia el fracaso.

De igual manera, el informante 4 expresa "ciertamente estoy de acuerdo con los cambios, pues soy una persona de fácil adaptación y de verdad todo cambio que sea para mejor considero que debe ser implementado, todo lo que sea para mejorar y lo vi como una buena iniciativa puesto que ahora había un nuevo patrono y debemos abocarnos a sus ideales". En general, todo cambio de requerir a los buenos procederes, al desarrollo de ciertos valores basados en el equilibrio, la armonía, con lo cual se beneficie la parte laboral y directiva, para lograr juntos el crecimiento de la organización con unos niveles de competitividad altamente consolidados.

Sobre la base de estos planteamientos, el investigador fija que los Cambios organizacionales emergen de la necesidad de despedazar con el equilibrio existente, para luego transformarlo en otro mucho más provecho, en este proceso de metamorfosis puede ser que en un principio como ya se dijo, las fuerzas deben romper con el equilibrio actual, interactuando permanentemente con otras fuerzas que tratan de oponerse, por ejemplo la resistencia al cambio, el miedo a cambiar, barreras comunicativas entre otras, es por ello que cuando una institución se plantea un cambio, debe involucrar una serie de tareas para poder minimizar esta interacción de fuerzas negativas.

\section{Propagación de incertidumbre}

Definitivamente, el gerente independientemente de la administración donde se desenvuelve (tanto del sector público 0 privado), será sin duda alguna un ser transcendental de cambio, por su responsabilidad en la toma de decisiones así como la manera de administrar, donde el éxito está condicionado necesariamente por el entorno, en función de su capacidad para manejarse en el ámbito donde labora.

En vista de antes expuesto, la planificación juega un papel fundamental en la gestión gerencial, esta permite prever y tomar decisiones oportunamente, pues quien no lo hace, corre el riesgo de malgastar la capacidad de competencia, donde en el peor de los casos de desaparecer en el mercado al transcurrir un cierto tiempo.

En relación a esto, la propagación de incertidumbre para Robbins (2004), se manifiesta dentro de una serie de factores que pueden encontrarse todas las incertidumbres que pueden influir o afectar el diseño de la estructura organizacional así como de igual forma sobre los niveles de estrés generados a nivel laboral, ocasionando una inestabilidad que puede desencadenar momentos de amenazas incesantes.

En este sentido, el autor enuncia que otras propagaciones causantes de la incertidumbre es el estrés terrorista ya que existen organizaciones donde se presentan enfrentamientos constantes de amenazas, afectando la labor realizada día a día por el talento humano como las infraestructura que puedan verse alterada por esas acciones ejecutadas por terceras personas ajenas a ésta "Estado".

Por otro lado, Blanch y Col. (2003) indican que los orígenes o propagaciones de incertidumbres se encuentran determinados por las numerosas demandas de situaciones corrientes que pueden surgir como amenazantes, importantes, focales, así como desafiantes, provocadoras, ante los contextos ostentados dentro de la organización.

Sobre la base de las ideas expuestas, estos autores revelan que se orientan estas difusiones en función de contextos específicos de escenarios laborales, donde se 
Denny J. Pereira B.- Doile E. Rios P.

pueden considerar las instalaciones tanto físicas, técnicas 0 de proceso, salariales, organizacionales, políticas, sociales entre otros aspectos que influyen en las tipologías del rol laboral, dirigido por la conflictividad, saturación, ambigüedad, negatividad y variedad.

Partiendo de lo mencionado por el Informante 3 donde revelaba que al momento de la restructuración "personalmente me sentí muy mal (...) en el sentido de que yo era una persona que ya estaba acostumbrada hacer mis planificaciones, a dirigir y ya con esta nueva empresa no se podía hacer eso, no sabíamos ni que rumbo llevábamos"; aunado lo señalado en "cada uno de nosotros los gerentes estábamos con muchas expectativas e interrogantes que queríamos aclarar".

Sin duda alguna, el investigador, considera que estas vivencias 0 experiencias son catalogadas como todas esas incertidumbres presentadas diariamente en organizaciones en procesos de restructuración ya que el gerente depende del funcionamiento de las acciones desarrolladas en función de talento humano que labora en las institución, es decir, es el impacto ocasionado en la conducta del trabajador sobre la ejecución de forma diaria de las tareas.

Al respecto es relevante considerar que en la actualidad el gerente se enfrenta a un entorno complejo e incierto, por lo que su éxito no tan solo dependerá de la atención que le preste a los indicadores financieros; aunque este siga siendo su foco, dado que las empresas globales o locales seguirán exigiendo cada vez más rentabilidad, pues, se ha de conocer su probabilidades de ocurrencia, atados a la relación existente a los distintos riesgos, para posteriormente establecer una estrategia de prevención, el cual contempla todos los elementos que contiene el entorno, incorporando en un sólo documento, tres elementos fundamentales, como lo son la inseguridad, miedo al cambio y barreras comunicacionales.

\section{HACIA UNA APROXIMACIÓN TEÓRICA SUSTANTIVA}

El objetivo de este apartado es expresar los hallazgos teóricos de la investigación, los cuales han de estar concebidos en una propuesta teórica - práctica orientada a explicar las interacciones que se producen de la acción del Outplacement como herramienta estratégica para reducir momentos de Incertidumbre en organizaciones, cuya misión principal tendrá repercusiones significativas en la transformación de los procesos organizacional. El presente estudio se realizará en diversas empresas que en sus inicios fueron privadas y pasaron a ser públicas como lo son la Red de Abastos Bicentenario S.A., PDVSA Gas S.A., Banco de Venezuela S.A., todas ubicadas en el municipio Maracaibo, estado Zulia de la República Bolivariana de Venezuela.
Asimismo, se hace necesario indicar que para la selección de los informantes clave se tomará en cuenta tanto a las personas activas en estas organizaciones como a las desvinculadas por este proceso de cambio de filosofía e ideología organizacional, deben ser trabajadores que puedan dar fe de las experiencias vividas y llenas de incertidumbres, con nivel académico mínimo de tercer nivel para garantizar una explicación más clara y profunda de los hechos narrados, así como seleccionar una muestra que incluya diferente nivel jerárquico en la estructura organizativa con la intención de conocer su robustez ante las acciones que desarrollan en su puesto de trabajo.

Con el desarrollo de esta propuesta, se ofrece respuesta a las siguientes interrogantes fenomenológicas: ¿De qué manera el outplacement puede reducir los momentos de incertidumbre generados en organizaciones de gestión transitoria de privada a pública? ¿Qué significado dan los trabajadores, según la experiencia vivida, al outplacement? ¿Cuáles actitudes se asumen ante una desvinculación?, al mismo tiempo, se articulan insumos teóricos, empíricos y prácticos para derivar un conjunto de proposiciones de entrada, los cuales surgen al vincular los aspectos desarrollados en las bases teóricas con relación a los hallazgos obtenidos en el estudio; de allí, que el abordaje teórico es realizado con base a los resultados alcanzados por cada una de las categorías que configuran el estudio sobre los cuales se desarrollaron elementos novedosos para la comunidad científica.

Esta propuesta desde la teoría pasa a ser la base para la conformación de una serie de fases sistematizadas, cumpliendo de acuerdo con el propósito central, desde este punto se parte para explicar la afinidad e interrelación que pudiese existir entre ambas categorías de estudio. No obstante, el interés no es adjudicarle dependencia a una respecto a la otra; sin embargo, es interesante comprender el outplacement como herramienta estratégica para la reducción de momentos de incertidumbre en organizaciones que han cambiado su filosofía organizacional. Asimismo, el pensamiento complejo que aviva desde las organizaciones, privilegia el abordaje de las realidades gerenciales desde el todo y desde cada una de sus partes sin despojarlas de la esencia de totalidad.

Para llevar a cabo el propósito del estudio, fue pertinente darle sentido teórico al conjunto de manifestaciones empíricas y anécdotas, que en torno al outplacement y a la incertidumbre, gravitaron en cada uno de los escenarios visitados. Por lo tanto, del análisis de los presupuestos teóricos que sirvieron como basamento referencial, conjuntamente con los hallazgos derivados de la investigación se genera un sistema demarcado por conceptos que imprimen significado a cada una de las acciones a establecerse. 
Ahora bien, para llevar a cabo lo anterior, resultó indispensable profundizar la investigación identificando las relaciones y vinculaciones entre los constructos teóricos objeto de estudio, así con los aspectos que condicionan su integración. Esto permitió la construcción de proposiciones donde se vinculan las cualidades y competencias del outplacement,así como la de la incertidumbre organizacional. Sobre la base de lo expresado, los resultados del presente proceso fenomenológico introspectivo - vivencial, se centraron de acuerdo a la estructuración de los propósitos de investigación, en las debilidades detectadas en los constructos y hechos manifestados.

A continuación, se presenta la propuesta integrada que concentra todos y cada uno de los elementos que emergieron de las entrevistas realizadas a los informantes, e incluso que sirva como referencia para desarrollar las teorías de entrada del presente estudio. Por tal motivo, a continuación se presentan la estructura conceptual final.

Figura 1: Estructura cognitiva integradora

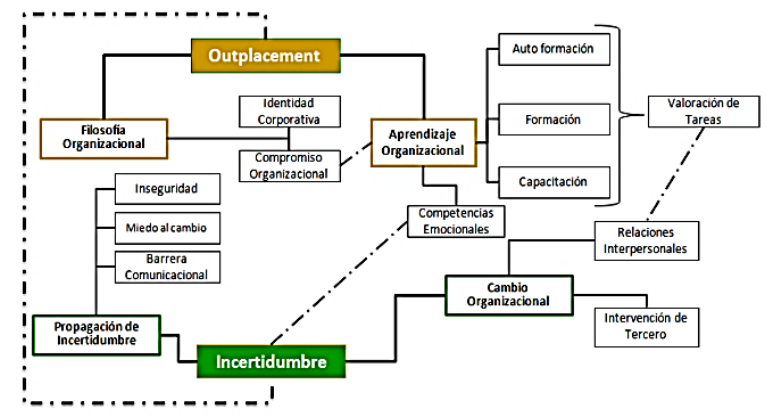

Fuente: Elaboración propia (2015)

El Outplacement es una práctica reforzadora que ayuda tanto a las organizaciones en proceso de cambio como a los empleados afectados. Es ideal en un proceso de cambio de filosofía organizacional pues proporcionar servicios diversificados de fácil adaptación a cada una de las necesidades de cada empleado, independientemente de su nivel de responsabilidad y afinidad.

La capacidad organizacional es un activo emprendedor, dinámico e innovador que demanda flexibilidad para adaptarse a las diferentes necesidades que se requiera, adquiriendo una identidad corporativa consolidada, manejándola como parte de un plan estratégico, convirtiendo en beneficio la minimización de la rotación laboral, mantiene la productividad y el compromiso hacia la organización, demostrando respeto con el talento reforzando significativamente su imagen ante sus empleados.
La implementación de esta herramienta desarrolla en las organizaciones programas de motivación, orientación y perfeccionamiento donde las personas implicadas siguen diferentes ciclos de aprendizaje, logrando la formación del individuo reorientándolo a la inserción laboral de colectivos con unas características propias, incentivándolos a la auto formación dirigidas a las disposiciones de la gobernabilidad corporativa en lugar de desafiar todo tipo de trabajos encomendados.

El papel de las organizaciones en el aprendizaje de sus empleados se extiende cada vez más; la capacitación corporativa se enfoca hacia el desarrollo del talento humano y sus relaciones interpersonales, para obtener resultados positivos concernientes a la valoración de las tareas asignadas por el proceso de cambio, vigorizando cada vez más sus competencias emocionales, previniendo así cualquier momento de incertidumbre.

La herramienta Outplacement trata de ayudar a enfrentar situaciones incomodas que puede ser traumática y propagadora de incertidumbres generando en el ambiente laboral inseguridades, miedo a cambiar y salir de la zona de confort, además de tener que luchar diariamente con barreras comunicacionales que prohíban expresarse libremente en lugar de trabajo, transformándolas en una fuente de oportunidades, considerarlo así el cambio como ventajoso.

En virtud de esto, el cambio organizacional muchas veces puede llegar a ser un impulso, para que algunos empleados mejoren en su desempeño profesional, relacionándose adecuadamente independientemente de una intervención de terceros y obligándolos a tomar las riendas de la flexibilidad en un contexto cambiante, alejándolos del conformismo que genera la rutina 0 costumbre laboral.

\section{MECANISMO OUTPLACEMENT}

Entre los propósitos de este estudio, esta generar una teoría sustantiva, sobre el outplacement como herramienta estratégica para reducir momentos de incertidumbre en organizaciones, pues desde este punto de vista, podrían las empresas utilizar dicha competencia resolutoria de conflictos emocionales existentes ante un cambio, donde el empleado desvinculado, pueda sentirse sumamente gratificado por el interés institucional manifestado hacia él, observando el nuevo escenario, no como un proceso rígido, duro y difícil, más bien, tomado como una oportunidad de experimentar nuevos desafíos profesionales.

Ahora bien, ello es posible, tanto para quien es asistido, como para el grupo de trabajadores que se encuentra en su entorno, llevando a cabo sus funciones. Debe 
Denny J. Pereira B.- Doile E. Rios P.

proyectarse el escenario del ex compañero a su propia realidad, haciéndoles pensar, que tal como fuera tratado, lo mismo puede ocurrir con todo el talento humano, logrando aumentar sentimientos de confianza, seguridad, valoración e identidad en la organización, lo cual sería percibido de manera positiva.

Sin duda alguna se puede señalar que el outplacement, representa una herramienta que logra obtener resultados satisfactorios en la desvinculación al actuar como un proceso de revisión, apoyo, asesoría, orientación, capacitación, dirigida a todas esas personas vistas por egresar o transferir, en la búsqueda incesante de un nuevo empleo, actividad, nivel y escenarios similares a las de su anterior ocupación, todo en el menor tiempo posible digerido 0 asimilado por el trabajador de manera satisfactoria.

Por ello, esta investigación, permitió al autor, tener conciencia plena de las necesidades existentes de una fase de sensibilización previa al proceso de outplacement: la primera, tiene que ver con el obstáculo emocional experimentado en situaciones de desprendimiento, estas generan alto nivel de incertidumbre. Además, atiende la auto - revisión, momento en el cual se asume con mayor rapidez la situación actual, generando así el aumento de posibilidades de reinserción organizacional.

Igualmente, la segunda fase, denominado valoración de competencias embarga la estimación de capacidades, destrezas, habilidades, conocimientos y aptitudes del personal desvinculado, esto permite conocer cuál o cuáles competencias se pueden potenciar al máximo en su ámbito laboral. Con esto, enriquecen el perfil profesional del asistido, realizando un análisis laboral actualizado.

Figura 2: Fases de sensibilización previa al proceso de outplacement.

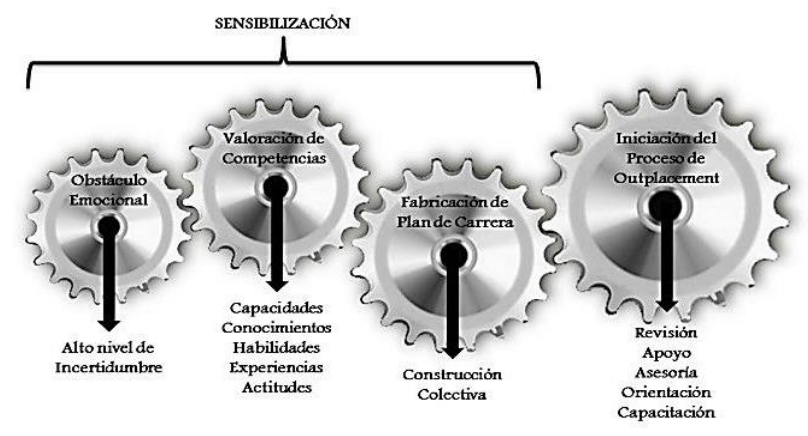

Fuente: Elaboración propia (2015)

Al mismo tiempo, teniendo todos estos datos como referencia, se desarrolla conjuntamente con el trabajador, la tercera fase, atendiendo la fabricación de un plan de carrera apropiado, donde se examinan las diferentes posibilidades exhibidas. Finalmente, cumpliendo estas fases, comenzaría la iniciación de la herramienta, recibiendo orientación, feed - back, respecto al desempeño en un proceso de revisión, apoyo, asesoría, orientación y capacitación en materia de selección de personal.

Resumiendo lo anterior, esta herramienta tiene como propósito, limitar en su justa medida momentos de incertidumbre, así como resolver de mejor forma escenarios traumáticos o estresantes. Transformándola en un pozo de oportunidades, para que algunos trabajadores mejoren significativamente su desempeño profesional, orientándolos a tomar su propio destino laboral y apartándoles del conformismo que genera la práctica rutinaria, abandonando de tal manera actitudes 0 acciones impulsivas, negativas e incluso nefastas.

\section{RESULTADOS Y CONCLUSIONES:}

Sobre la base de los resultados obtenidos, producto de la información expuesta en las líneas previas confrontada con las teorías abordadas en la investigación y en función del cumplimiento de los propósitos formulados, se derivan las siguientes conclusiones:

El Outplacement ayuda al talento humano a encontrar un empleo y/o actividad que se adecue más a su perfil laboral y optar por un cambio que les sea significativo en su vida profesional. Hoy en día se pudo constatar que las instituciones en procesos desvinculativos, se encuentran pocos dispuestos a implementar un rol facilitador, imposibilitando en el afectado la pronta reinserción laboral y social de sus ex empleados.

Frente a esta realidad, el Outplacement puede efectuar una efectiva contribución a las personas asistidas, se corroboró así la inexistencias de técnicas o programas que permitan generar estrategias fortalecedoras o bien para encontrar un nuevo empleo y/o nuevas modalidades de emprendimiento. Ciertamente, el método de desvinculación asistida generar servicios o habilidades propios para un trabajo adecuado.

Sin embargo las organizaciones actualmente no enseñan a su personar a aceptar, asumir y dar frente a esta nueva realidad de forma adaptativa en un mundo abierto a la incertidumbre, pues no desarrollan habilidades y servicios útiles y/o necesarios a otras personas 0 al mercado en sí.

En definitiva, se plantea que el Outplacement es un proceso con una metodología y técnicas propias que tienen en cuenta las características del individuo y del mercado laboral en el que se encuentra. Se conoció que las empresas no gestionan adecuadamente la transformación 
de un proceso de reestructuración, limitando a las personas una oportunidad de cambio, y una adecuación mejora de los recursos humanos de la organización.

En este ámbito, la herramienta se emplea no sólo para quienes son egresados sino también para quienes deseen desarrollarse profesionalmente de forma distinta 0 simplemente mejorar sus condiciones de empleo mediante un cambio significativo de empleador. Se reconoció que las organizaciones aplican dicha herramienta en desvinculaciones asistidas de alto nivel estructural, es decir cuando se trata de niveles gerenciales.

Finalmente, Las organizaciones comprometidas con este tipo de herramientas estratégicas experimentan importantes beneficios los cual se ven reflejados no solo en los empleados que han sido desvinculados, sino también en los que siguen formando parte de la organización, incrementando la motivación y el compromiso, mejorando la comunicación interna, aumentando el sentido de pertenencia y reforzando la cultura organizacional.

De allí que por consiguiente, una vez presentados estos puntos concluyentes, se logró comprender el outplacement como herramienta estratégica para reducir momentos de incertidumbre en organizaciones en proceso de transición y cambio de filosofía organizacional, encontrándose la inexistencias de técnicas o programas para tales fines, tal como se describió en cada una de las conclusiones anteriores.

\section{REFERENCIAS:}

Barragán, J (2000) Outplacement. Tomado de http://www.uaq.mx/psicologia (17 de enero de 2016)

Barnes S. (2006).Sistemas de Gestión del Conocimiento. Thompson Editores Spain. España.

Blanch, J., Espuny, M., Gala, C., y Martín, A. (2003). Teoría de las relaciones laborales. Fundamentos. Editorial UOC. España.

Carrión, J. (2005). Elementos de Cambio. Editorial La Muralla. Madrid.

Carmona, M. (2009). El Conocimiento Personal. Un valor Seguro. Gestione su Conocimiento y Saque Partido. Prentice Hall. España.

Chiavenato, I. (2007). Administración de recursos humanos. Mc Graw Hill. Colombia.
Denisson, E. (2006). Modelo de gestión de seguridad. Editorial Silva. España.

Drucker, P. (2005). 366 días de reflexiones claves para acertar en sus negocios. Editorial Granica, primera edición. Barcelona, España.

Fernandez, J. (2004). Arquitectura del cambio organizacional. II parte. Word Wide web: www.gestiopolis.com (20 de octubre de 2015)

Folk R, Sánchez, G, Vallbe, C. (2006) Aprendizaje organizacional. Un nuevo reto para Recursos Humanos. Xarxa Consultors www.educaweb.com/ (27 de diciembre de 2015)

León, A. (2006), estrategias para el desarrollo de la comunicación profesional, segunda edición, editorial Limusa. México D.F

León O. (2006). Tomar Decisiones difíciles. Segunda Edición. Editorial Mc Graw Hill. Madrid (España).

Licht, A. (2003) ¿Qué es el Outplacement? Entrevista tomada Word Wide web: www.rrhhmagazine.com (19 de octubre de 2016)

Muñiz, R. (2008). Estrategia de Marketing y la Dirección Estratégica. Editorial Mc Graw Hill, tercera edición. México.

Pérez, C. (2008). Pensamiento Estratégico. Unidad III. Fundamentos del pensamiento estratégico. Material de apoyo. "Universidad Rafael Belloso Chacín". Maracaibo, Venezuela.

Robbins, S. (2004) Comportamiento organizacional. Prentice Hall editores. México.

Sastre, L (1995). La desvinculación asistida, (outplacement) y la continuidad en el empleo: Volumen 6 Revista de Psicología. Universidad de Chile. Chile.

Senge, P. (2005). La Quinta disciplina. El Arte y la práctica de la organización alerta al aprendizaje. Editorial Granica. Buenos Aires. Argentina.

Strauss, A. y Corbin, J. (2002) Bases de la investigación cualitativa. Técnicas y procedimientos para desarrollar la teoría fundamentada. (2da. Edición). CONTUS-editorial Universidad de Antioquia. Bogotá-Colombia. 
OUTPLACEMENT COMO HERRAMIENTA ESTRATÉGICA PARA REDUCIR MOMENTOS DE INCERTIDUMBRE EN ORGANIZACIONES: UNA PERSPECTIVA REFLEXIVA Y ATEMPORAL

Denny J. Pereira B.- Doile E. Rios P.

Sull D. y Escolari M.(2005). Success against the odds: what brazilian champions teach us about thriving in un predictable markets. Editorial Elsevier. Brasil.

Swieringa, J. y Wierdsma, E. (2007). Las Organizaciones que Aprenden. Editorial. México. 